\title{
Exploring Deep-UV Nonlinear Optical Materials with Enhanced \\ Second Harmonic Generation Response and Birefringence in \\ Fluoroaluminoborate Crystals
}

\author{
Hongkun Liu, ${ }^{\mathrm{a}}$ Bingbing Zhang, ${ }^{\mathrm{a}}$ Ling Li, ${ }^{\mathrm{b}}$ and Ying Wang* ${ }^{\mathrm{a}}$ \\ ${ }^{a}$ College of Chemistry and Environmental Science, Hebei University, Baoding 071002, \\ China.Email: wangy@hbu.edu.cn. \\ ${ }^{b}$ College of Physics Science and Technology, Hebei University, Baoding 071002, \\ China.
}

\section{Table of Contents}

Table S1. Known fluoroaluminoborate NLO crystals.

Table S2. Atomic coordinates and equivalent isotropic displacement parameters for RABF and CRABF.......

Table S3. Selected bond distances $(\AA)$ and angles (deg) of RABF.

Table S4. Selected bond distances and angles $(\AA)$ of CRABF.

Figure S1. Crystal structure comparison of (a) $\mathrm{CsB}_{4} \mathrm{O}_{6} \mathrm{~F}$, (b) $\mathrm{CsAlB}_{3} \mathrm{O}_{6} \mathrm{~F}$, (c) $\mathrm{RbB}_{4} \mathrm{O}_{6} \mathrm{~F}$, (d) $\mathrm{RABF}$, (e) $\mathrm{Cs}_{0.5} \mathrm{Rb}_{0.5} \mathrm{~B}_{4} \mathrm{O}_{6} \mathrm{~F}$, and (f) $\mathrm{CRABF}$.

Figure S2. The calculated and experiment PXRD patterns of (a) RABF and (b) CRABF.............S9

Figure S3. EDX spectroscopy and SEM image (insets) of (a) RABF and (b) CRABF................S10

Figure S4. The calculated and experiment PXRD patterns of $\mathrm{Cs}_{x} \mathrm{Rb}_{1-x} \mathrm{AlB}_{3} \mathrm{O}_{6} \mathrm{~F}$ with different $x$ values.

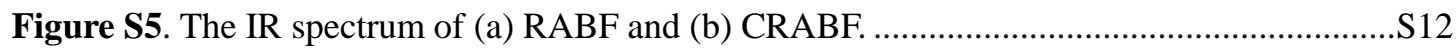

Figure S6. The TG/DSC curves of (a) RABF and (b) CRABF. ............................................... 13

Figure S7. The XRD pattarns of before and after melting of (a) RABF and (b) CRABF. ...........S14

Figure S8. The diffuse reflectance spectrum of (a) RABF and (b) CRABF...............................S15

Figure S9. The SHG intensities of $\mathrm{Cs}_{x} \mathrm{Rb}_{1-x} \mathrm{AlB}_{3} \mathrm{O}_{6} \mathrm{~F}$ with different $x$ values. .............................. 16

Figure S10. The band gap and PDOS of (a-b) RABF and (c-d) CRABF. .................................... 17

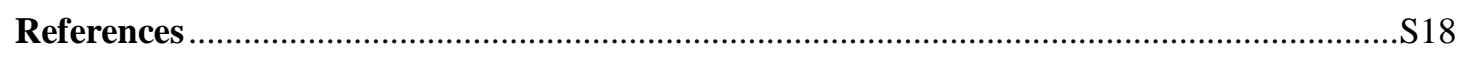


Table S1. Known fluoroaluminoborate NLO crystals.

\begin{tabular}{|c|c|c|c|c|c|c|}
\hline Crystals & $\begin{array}{l}\text { Space } \\
\text { group }\end{array}$ & FBUs & $\begin{array}{l}\text { Cutoff } \\
\text { edge } \\
(\mathrm{nm})\end{array}$ & $\begin{array}{l}\text { PSHG } \\
(\times \mathrm{KDP})\end{array}$ & $\begin{array}{l}\text { Birefringe } \\
\text { nce (calc.) }\end{array}$ & $\begin{array}{l}\text { Shortest } \\
\text { phase-matchin } \\
\mathrm{g} \text { wavelength } \\
(\mathrm{nm})\end{array}$ \\
\hline $\mathrm{BaAlBO}_{3} \mathrm{~F}_{2}{ }^{1,2}$ & $P \overline{6} 2 c$ & $\mathrm{BO}_{3} / \mathrm{AlO}_{4} \mathrm{~F}_{2}$ & 165 & 2 & $\begin{array}{l}0.042 @ 10 \\
64 \text { nm }\end{array}$ & 273 \\
\hline $\mathrm{BaAl}_{1-\mathrm{x}} \mathrm{Ga}_{\mathrm{x}} \mathrm{BO}_{3} \mathrm{~F}_{2}{ }^{3}$ & $P \overline{6} 2 c$ & $\begin{array}{l}\mathrm{BO}_{3} / \mathrm{Al}(\mathrm{Ga}) \\
\mathrm{O}_{4} \mathrm{~F}_{2}\end{array}$ & 265 & 2 & $\begin{array}{l}0.0442 @ 1 \\
064 \text { nm }\end{array}$ & N/A \\
\hline $\mathrm{Rb}_{3} \mathrm{Al}_{3} \mathrm{~B}_{3} \mathrm{O}_{10} \mathrm{~F}^{4}$ & $P 3_{1} \mathrm{c}$ & $\begin{array}{l}\mathrm{BO}_{3} / \mathrm{AlO}_{4} / \\
\mathrm{AlO}_{3} \mathrm{~F}\end{array}$ & $\sim 200$ & 1.2 & N/A & N/A \\
\hline $\mathrm{CsAlB}_{3} \mathrm{O}_{6} \mathrm{~F}^{5}$ & Pna $_{1}$ & $\mathrm{~B}_{3} \mathrm{O}_{6} / \mathrm{AlO}_{3} \mathrm{~F}$ & $\begin{array}{l}<190 \\
166 \\
\text { (calc.) }\end{array}$ & 2 & $\begin{array}{l}0.091 \\
@ 1064 \\
\mathrm{~nm}\end{array}$ & 182 (calc.) \\
\hline
\end{tabular}

N/A = Not available. 
Table S2. Atomic coordinates and equivalent isotropic displacement parameters for RABF and CRABF.

\begin{tabular}{|c|c|c|c|c|c|c|}
\hline Atom & Wyck & $\mathbf{x} / a$ & $\mathrm{y} / b$ & $\mathrm{z} / c$ & $U_{\text {eq }}^{[\mathrm{a}]}$ & BVS \\
\hline \multicolumn{7}{|c|}{$\mathrm{RbAlB}_{3} \mathrm{O}_{6} \mathrm{~F}$} \\
\hline $\mathrm{Rb}(1)$ & $4 a$ & $0.3864(1)$ & $0.5981(1)$ & $0.5405(2)$ & $0.022(1)$ & 1.16 \\
\hline $\mathrm{Rb}(2)$ & $4 a$ & $0.7740(1)$ & $0.2520(1)$ & $0.9725(2)$ & $0.022(1)$ & 1.26 \\
\hline $\mathrm{Rb}(3)$ & $4 a$ & $0.5662(1)$ & $-0.0878(1)$ & $0.5304(2)$ & $0.024(1)$ & 1.10 \\
\hline $\operatorname{Al}(1)$ & $4 a$ & $0.5794(2)$ & $0.5751(2)$ & $0.5313(7)$ & $0.019(1)$ & 3.09 \\
\hline $\operatorname{Al}(2)$ & $4 a$ & $0.5968(2)$ & $0.0925(2)$ & $1.0429(7)$ & $0.020(1)$ & 3.13 \\
\hline $\operatorname{Al}(3)$ & $4 a$ & $0.7363(2)$ & $0.0786(3)$ & $0.4756(6)$ & $0.019(1)$ & 3.03 \\
\hline $\mathrm{B}(1)$ & $4 a$ & $0.6095(8)$ & $0.7115(12)$ & $-0.1630(20)$ & $0.026(4)$ & 3.07 \\
\hline $\mathrm{B}(2)$ & $4 a$ & $0.6104(7)$ & $0.8573(10)$ & $0.0690(20)$ & $0.022(4)$ & 3.09 \\
\hline $\mathrm{B}(3)$ & $4 a$ & $0.6053(8)$ & $0.6690(13)$ & $0.1680(20)$ & $0.022(4)$ & 2.99 \\
\hline $\mathrm{B}(4)$ & $4 a$ & $0.5842(7)$ & $0.3433(11)$ & $0.5780(20)$ & $0.021(4)$ & 3.10 \\
\hline $\mathrm{B}(5)$ & $4 a$ & $0.5541(8)$ & $0.1987(11)$ & $0.3690(20)$ & $0.019(4)$ & 3.05 \\
\hline $\mathrm{B}(6)$ & $4 a$ & $0.5574(8)$ & $0.1665(12)$ & $0.6990(20)$ & $0.022(4)$ & 3.08 \\
\hline $\mathrm{B}(7)$ & $4 a$ & $0.7657(7)$ & $-0.0053(10)$ & $0.8286(19)$ & $0.013(3)$ & 3.09 \\
\hline $\mathrm{B}(8)$ & $4 a$ & $0.7588(7)$ & $-0.1900(12)$ & $0.9270(20)$ & $0.019(4)$ & 3.03 \\
\hline $\mathrm{B}(9)$ & $4 a$ & $0.7689(7)$ & $-0.0511(11)$ & $1.1570(20)$ & $0.018(3)$ & 3.00 \\
\hline $\mathrm{O}(1)$ & $4 a$ & $0.6109(4)$ & $0.6801(6)$ & $-0.3361(11)$ & $0.021(2)$ & 2.14 \\
\hline $\mathrm{O}(2)$ & $4 a$ & $0.6145(5)$ & $0.8190(6)$ & $-0.1144(12)$ & $0.024(2)$ & 2.06 \\
\hline $\mathrm{O}(3)$ & $4 a$ & $0.6104(4)$ & $0.9608(6)$ & $0.1089(12)$ & $0.032(3)$ & 2.10 \\
\hline $\mathrm{O}(4)$ & $4 a$ & $0.6076(5)$ & $0.7806(6)$ & $0.2075(12)$ & $0.026(2)$ & 2.13 \\
\hline $\mathrm{O}(5)$ & $4 a$ & $0.6058(4)$ & $0.6365(6)$ & $-0.0149(13)$ & $0.028(2)$ & 2.06 \\
\hline $\mathrm{O}(6)$ & $4 a$ & $0.6008(4)$ & $0.5944(6)$ & $0.3022(12)$ & $0.027(2)$ & 2.01 \\
\hline $\mathrm{O}(7)$ & $4 a$ & $0.5988(4)$ & $0.4463(6)$ & $0.6083(12)$ & $0.032(3)$ & 2.02 \\
\hline $\mathrm{O}(8)$ & $4 a$ & $0.5765(5)$ & $0.3033(6)$ & $0.4002(13)$ & $0.032(3)$ & 2.06 \\
\hline $\mathrm{O}(9)$ & $4 a$ & $0.5505(4)$ & $0.1555(7)$ & $0.2032(12)$ & $0.029(2)$ & 2.10 \\
\hline $\mathrm{O}(10)$ & $4 a$ & $0.5364(3)$ & $0.1400(6)$ & $0.5246(12)$ & $0.022(2)$ & 2.13 \\
\hline $\mathrm{O}(11)$ & $4 a$ & $0.5784(4)$ & $0.2725(6)$ & $0.7232(12)$ & $0.026(2)$ & 2.15 \\
\hline $\mathrm{O}(12)$ & $4 a$ & $0.5574(4)$ & $0.0962(6)$ & $0.8356(11)$ & $0.024(2)$ & 2.16 \\
\hline $\mathrm{O}(13)$ & $4 a$ & $0.7656(4)$ & $0.0695(6)$ & $0.6976(11)$ & $0.023(2)$ & 2.10 \\
\hline $\mathrm{O}(14)$ & $4 a$ & $0.7706(3)$ & $0.0249(5)$ & $0.10154(13)$ & $0.019(2)$ & 2.14 \\
\hline $\mathrm{O}(15)$ & $4 a$ & $0.7661(5)$ & $-0.0231(6)$ & $1.3366(12)$ & $0.026(2)$ & 2.07 \\
\hline$O(16)$ & $4 a$ & $0.7631(4)$ & $-0.1600(6)$ & $1.1097(12)$ & $0.024(2)$ & 2.13 \\
\hline $\mathrm{O}(17)$ & $4 a$ & $0.7629(4)$ & $-0.1135(6)$ & $0.7880(12)$ & $0.021(2)$ & 2.25 \\
\hline $\mathrm{O}(18)$ & $4 a$ & $0.7506(4)$ & $-0.2954(5)$ & $0.8795(12)$ & $0.025(2)$ & 1.99 \\
\hline $\mathrm{F}(1)$ & $4 a$ & $0.5059(3)$ & $0.5869(7)$ & $0.5429(15)$ & $0.058(2)$ & 0.99 \\
\hline $\mathrm{F}(2)$ & $4 a$ & $0.6589(3)$ & $0.1673(5)$ & $1.0243(12)$ & $0.038(2)$ & 0.88 \\
\hline $\mathrm{F}(3)$ & $4 a$ & $0.6630(3)$ & $0.0571(6)$ & $0.4942(12)$ & $0.050(2)$ & 0.88 \\
\hline \multicolumn{7}{|c|}{$\mathrm{Cs}_{0.5} \mathrm{Rb}_{0.5} \mathrm{AlB}_{3} \mathrm{O}_{6} \mathrm{~F}$} \\
\hline $\operatorname{Cs}(1)$ & $2 a$ & -1 & 0 & $-1 / 2$ & $0.059(1)$ & 0.85 \\
\hline
\end{tabular}


Supporting Information

\begin{tabular}{ccccccc}
$\mathrm{Rb}(1)$ & $2 a$ & -1 & 0 & $-1 / 2$ & $0.059(1)$ & 0.85 \\
$\mathrm{Al}(1)$ & $2 d$ & -1.33333 & $-2 / 3$ & $-3 / 4$ & $0.058(2)$ & 3.08 \\
$\mathrm{~B}(1)$ & $12 i$ & $-0.8860(40)$ & $-0.500(30)$ & $-0.7060(30)$ & $0.039(9)$ & 3.03 \\
$\mathrm{O}(1)$ & $12 i$ & $-0.8296(15)$ & $-0.2790(15)$ & $-0.6909(13)$ & $0.038(4)$ & 2.02 \\
$\mathrm{O}(2)$ & $12 i$ & $-1.0983(17)$ & $-0.6610(17)$ & $-0.7984(13)$ & $0.040(5)$ & 2.10 \\
$\mathrm{~F}(1)$ & $4 f$ & -1.33333 & $-2 / 3$ & $-0.5191(19)$ & $0.200(20)$ & 0.85 \\
& & & & & & \\
\hline
\end{tabular}

${ }^{[a]} U_{\text {eq }}$ is defined as one-third of the trace of the orthogonalized $U_{\mathrm{ij}}$ tensor. 
Table S3. Selected bond distances ( $\mathrm{A})$ and angles (deg.) of RABF.

\begin{tabular}{|c|c|c|c|}
\hline $\mathrm{Rb}(1)-\mathrm{O}(5) \# 1$ & $2.921(7)$ & $\mathrm{F}(1)-\mathrm{Al}(1)-\mathrm{O}(1) \# 12$ & $108.0(5)$ \\
\hline $\mathrm{Rb}(1)-\mathrm{O}(6) \# 1$ & $3.042(8)$ & $\mathrm{F}(1)-\mathrm{Al}(1)-\mathrm{O}(6)$ & $108.1(6)$ \\
\hline $\mathrm{Rb}(1)-\mathrm{O}(7) \# 2$ & $3.179(9)$ & $\mathrm{F}(1)-\mathrm{Al}(1)-\mathrm{O}(7)$ & $108.2(5)$ \\
\hline $\mathrm{Rb}(1)-\mathrm{O}(8) \# 1$ & $2.980(9)$ & $\mathrm{O}(6)-\mathrm{Al}(1)-\mathrm{O}(1) \# 12$ & $107.8(4)$ \\
\hline $\mathrm{Rb}(1)-\mathrm{O}(11) \# 2$ & $2.896(8)$ & $\mathrm{O}(6)-\mathrm{Al}(1)-\mathrm{O}(7)$ & $111.1(5)$ \\
\hline $\mathrm{Rb}(1)-\mathrm{O}(15) \# 3$ & $3.205(10)$ & $\mathrm{O}(7)-\mathrm{Al}(1)-\mathrm{O}(1) \# 12$ & $113.5(5)$ \\
\hline $\mathrm{Rb}(1)-\mathrm{O}(17) \# 4$ & $3.298(9)$ & $\mathrm{F}(2)-\mathrm{Al}(2)-\mathrm{O}(3) \# 8$ & $113.2(4)$ \\
\hline $\mathrm{Rb}(1)-\mathrm{F}(1)$ & $2.679(7)$ & $\mathrm{F}(2)-\mathrm{Al}(2)-\mathrm{O}(9) \# 12$ & $107.7(4)$ \\
\hline $\mathrm{Rb}(1)-\mathrm{F}(2) \# 2$ & $3.063(6)$ & $\mathrm{F}(2)-\mathrm{Al}(2)-\mathrm{O}(12)$ & $109.9(5)$ \\
\hline $\mathrm{Rb}(2)-\mathrm{O}(1) \# 5$ & $3.056(9)$ & $\mathrm{O}(3) \# 8-\mathrm{Al}(2)-\mathrm{O}(9) \# 12$ & $110.1(5)$ \\
\hline $\mathrm{Rb}(2)-\mathrm{O}(4) \# 5$ & $3.288(10)$ & $\mathrm{O}(3) \# 8-\mathrm{Al}(2)-\mathrm{O}(12)$ & $110.7(4)$ \\
\hline $\mathrm{Rb}(2)-\mathrm{O}(13)$ & $3.002(8)$ & $\mathrm{O}(12)-\mathrm{Al}(2)-\mathrm{O}(9) \# 12$ & $104.9(5)$ \\
\hline $\mathrm{Rb}(2)-\mathrm{O}(14)$ & $2.814(6)$ & $\mathrm{F}(3)-\mathrm{Al}(3)-\mathrm{O}(13)$ & $106.8(5)$ \\
\hline $\mathrm{Rb}(2)-\mathrm{O}(15) \# 6$ & $3.070(8)$ & $\mathrm{F}(3)-\mathrm{Al}(3)-\mathrm{O}(15) \# 13$ & $108.1(5)$ \\
\hline $\mathrm{Rb}(2)-\mathrm{O}(16) \# 6$ & $2.949(9)$ & $\mathrm{F}(3)-\mathrm{Al}(3)-\mathrm{O}(18) \# 6$ & $110.0(5)$ \\
\hline $\mathrm{Rb}(2)-\mathrm{O}(17) \# 7$ & $2.931(8)$ & $\mathrm{O}(13)-\mathrm{Al}(3)-\mathrm{O}(15) \# 13$ & $109.8(4)$ \\
\hline $\mathrm{Rb}(2)-\mathrm{O}(18) \# 7$ & $3.040(9)$ & $\mathrm{O}(18) \# 6-\mathrm{Al}(3)-\mathrm{O}(13)$ & $111.4(4)$ \\
\hline $\mathrm{Rb}(2)-\mathrm{F}(2)$ & $2.806(6)$ & $\mathrm{O}(18) \# 6-\mathrm{Al}(3)-\mathrm{O}(15) \# 13$ & $110.6(5)$ \\
\hline $\mathrm{Rb}(3)-\mathrm{O}(1) \# 8$ & $3.177(8)$ & $\mathrm{O}(1)-\mathrm{B}(1)-\mathrm{O}(2)$ & $121.9(13)$ \\
\hline $\mathrm{Rb}(3)-\mathrm{O}(2) \# 8$ & $3.006(9)$ & $\mathrm{O}(1)-\mathrm{B}(1)-\mathrm{O}(5)$ & $122.0(12)$ \\
\hline $\mathrm{Rb}(3)-\mathrm{O}(3) \# 9$ & $3.250(9)$ & $\mathrm{O}(2)-\mathrm{B}(1)-\mathrm{O}(5)$ & $116.1(13)$ \\
\hline $\mathrm{Rb}(3)-\mathrm{O}(4) \# 9$ & $2.983(8)$ & $\mathrm{O}(3)-\mathrm{B}(2)-\mathrm{O}(2)$ & $122.3(12)$ \\
\hline $\mathrm{Rb}(3)-\mathrm{O}(9) \# 10$ & $3.014(10)$ & $\mathrm{O}(3)-\mathrm{B}(2)-\mathrm{O}(4)$ & $120.7(13)$ \\
\hline $\mathrm{Rb}(3)-\mathrm{O}(10)$ & $2.882(7)$ & $\mathrm{O}(4)-\mathrm{B}(2)-\mathrm{O}(2)$ & 117.1(9) \\
\hline $\mathrm{Rb}(3)-\mathrm{O}(12)$ & $3.162(8)$ & $\mathrm{O}(5)-\mathrm{B}(3)-\mathrm{O}(4)$ & $118.7(13)$ \\
\hline $\mathrm{Rb}(3)-\mathrm{O}(12) \# 11$ & $3.107(9)$ & $\mathrm{O}(6)-\mathrm{B}(3)-\mathrm{O}(4)$ & $121.8(13)$ \\
\hline $\mathrm{Rb}(3)-\mathrm{F}(3)$ & $2.820(7)$ & $\mathrm{O}(6)-\mathrm{B}(3)-\mathrm{O}(5)$ & $119.5(12)$ \\
\hline $\mathrm{Al}(1)-\mathrm{O}(1) \# 12$ & $1.755(9)$ & $\mathrm{O}(7)-\mathrm{B}(4)-\mathrm{O}(8)$ & $121.3(12)$ \\
\hline $\mathrm{Al}(1)-\mathrm{O}(6)$ & $1.735(10)$ & $\mathrm{O}(7)-\mathrm{B}(4)-\mathrm{O}(11)$ & $120.6(12)$ \\
\hline $\mathrm{Al}(1)-\mathrm{O}(7)$ & $1.735(8)$ & $\mathrm{O}(11)-\mathrm{B}(4)-\mathrm{O}(8)$ & $118.0(11)$ \\
\hline $\mathrm{Al}(1)-\mathrm{F}(1)$ & $1.656(8)$ & $\mathrm{O}(9)-\mathrm{B}(5)-\mathrm{O}(8)$ & $122.7(13)$ \\
\hline $\mathrm{Al}(2)-\mathrm{O}(3) \# 8$ & $1.717(8)$ & $\mathrm{O}(9)-\mathrm{B}(5)-\mathrm{O}(10)$ & $120.4(12)$ \\
\hline $\mathrm{Al}(2)-\mathrm{O}(9) \# 12$ & $1.736(10)$ & $\mathrm{O}(10)-\mathrm{B}(5)-\mathrm{O}(8)$ & $116.9(12)$ \\
\hline $\mathrm{Al}(2)-\mathrm{O}(12)$ & $1.735(10)$ & $\mathrm{O}(10)-\mathrm{B}(6)-\mathrm{O}(11)$ & $116.7(12)$ \\
\hline $\operatorname{Al}(2)-F(2)$ & $1.673(7)$ & $\mathrm{O}(12)-\mathrm{B}(6)-\mathrm{O}(10)$ & $121.8(12)$ \\
\hline $\mathrm{Al}(3)-\mathrm{O}(13)$ & $1.733(9)$ & $\mathrm{O}(12)-\mathrm{B}(6)-\mathrm{O}(11)$ & $121.6(14)$ \\
\hline $\mathrm{Al}(3)-\mathrm{O}(15)$ & $1.737(9)$ & $\mathrm{O}(13)-\mathrm{B}(7)-\mathrm{O}(14)$ & $120.2(10)$ \\
\hline $\mathrm{Al}(3)-\mathrm{O}(18) \# 6$ & $1.723(8)$ & $\mathrm{O}(13)-\mathrm{B}(7)-\mathrm{O}(17)$ & $121.9(12)$ \\
\hline $\mathrm{Al}(3)-\mathrm{F}(3)$ & $1.669(8)$ & $\mathrm{O}(17)-\mathrm{B}(7)-\mathrm{O}(14)$ & $117.9(10)$ \\
\hline $\mathrm{B}(1)-\mathrm{O}(1)$ & $1.305(18)$ & $\mathrm{O}(16)-\mathrm{B}(8)-\mathrm{O}(17)$ & $120.5(12)$ \\
\hline $\mathrm{B}(1)-\mathrm{O}(2)$ & $1.374(17)$ & $\mathrm{O}(18)-\mathrm{B}(8)-\mathrm{O}(16)$ & $120.6(12)$ \\
\hline $\mathrm{B}(1)-\mathrm{O}(5)$ & $1.415(18)$ & $\mathrm{O}(18)-\mathrm{B}(8)-\mathrm{O}(17)$ & $118.8(12)$ \\
\hline
\end{tabular}




\begin{tabular}{llll}
\hline $\mathrm{B}(2)-\mathrm{O}(2)$ & $1.406(18)$ & $\mathrm{O}(14)-\mathrm{B}(9)-\mathrm{O}(16)$ & $118.3(11)$ \\
$\mathrm{B}(2)-\mathrm{O}(3)$ & $1.306(14)$ & $\mathrm{O}(15)-\mathrm{B}(9)-\mathrm{O}(14)$ & $122.6(11)$ \\
$\mathrm{B}(2)-\mathrm{O}(4)$ & $1.375(16)$ & $\mathrm{O}(15)-\mathrm{B}(9)-\mathrm{O}(16)$ & $118.7(11)$ \\
$\mathrm{B}(3)-\mathrm{O}(4)$ & $1.404(17)$ & & \\
$\mathrm{B}(3)-\mathrm{O}(5)$ & $1.375(18)$ & & \\
$\mathrm{B}(3)-\mathrm{O}(6)$ & $1.339(18)$ & & \\
$\mathrm{B}(4)-\mathrm{O}(7)$ & $1.327(15)$ & & \\
$\mathrm{B}(4)-\mathrm{O}(8)$ & $1.387(17)$ & & \\
$\mathrm{B}(4)-\mathrm{O}(11)$ & $1.365(17)$ & & \\
$\mathrm{B}(5)-\mathrm{O}(8)$ & $1.399(16)$ & & \\
$\mathrm{B}(5)-\mathrm{O}(9)$ & $1.391(17)$ & & \\
$\mathrm{B}(5)-\mathrm{O}(10)$ & $1.312(17)$ & & \\
$\mathrm{B}(6)-\mathrm{O}(10)$ & $1.382(18)$ & & \\
$\mathrm{B}(6)-\mathrm{O}(11)$ & $1.397(17)$ & & \\
$\mathrm{B}(6)-\mathrm{O}(12)$ & $1.311(17)$ & & \\
$\mathrm{B}(7)-\mathrm{O}(13)$ & $1.319(15)$ & & \\
$\mathrm{B}(7)-\mathrm{O}(14)$ & $1.401(17)$ & & \\
$\mathrm{B}(7)-\mathrm{O}(17)$ & $1.365(14)$ & & \\
$\mathrm{B}(8)-\mathrm{O}(16)$ & $1.373(17)$ & & \\
$\mathrm{B}(8)-\mathrm{O}(17)$ & $1.376(16)$ & & \\
$\mathrm{B}(8)-\mathrm{O}(18)$ & $1.354(16)$ & & \\
$\mathrm{B}(9)-\mathrm{O}(14)$ & $1.384(15)$ & & \\
$\mathrm{B}(9)-\mathrm{O}(15)$ & $1.342(16)$ & & \\
$\mathrm{B}(9)-\mathrm{O}(16)$ & $1.389(15)$ & & \\
\hline $\mathrm{S} y m+10$ & & \\
\end{tabular}

Symmetry transformations used to generate equivalent atoms:

\#1 $\quad 1-\mathrm{x}, 1-\mathrm{y}, 0.5+\mathrm{z} ; \quad \# 2 \quad$ 1-x,1-y,-0.5+z; \#3 $\quad-0.5+\mathrm{x}, 0.5-\mathrm{y},-1+\mathrm{z} ; \quad \# 4 \quad-0.5+\mathrm{x}, 0.5-\mathrm{y}, \mathrm{z} ;$

\#5 $\quad 1.5-\mathrm{x},-0.5+\mathrm{y}, 1.5+\mathrm{z} ; \quad \# 6 \quad 1.5-\mathrm{x}, 0.5+\mathrm{y},-0.5+\mathrm{z} ; \quad \# 7 \quad 1.5-\mathrm{x}, 0.5+\mathrm{y}, 0.5+\mathrm{z} ; \quad \# 8 \quad \mathrm{x},-1+\mathrm{y}, 1+\mathrm{z}$;

\#9 $\quad \mathrm{x},-1+\mathrm{y}, \mathrm{z} ; \quad \# 10 \quad 1-\mathrm{x},-\mathrm{y}, 0.5+\mathrm{z} ; \quad \# 111-\mathrm{x},-\mathrm{y},-0.5+\mathrm{z} ; \quad \# 12 \quad \mathrm{x}, \mathrm{y}, 1+\mathrm{z} ; \quad \# 13 \quad \mathrm{x}, \mathrm{y}-1+\mathrm{z}$ 
Table S4. Selected bond distances ( $(\AA)$ and angles (deg.) of CRABF.

\begin{tabular}{llll}
\hline $\mathrm{Cs}(1) / \mathrm{Rb}(1)-\mathrm{O}(1)$ & $3.145(11)$ & $\mathrm{O}(2)-\mathrm{Al}(1)-\mathrm{F}(1)$ & $103.5(4)$ \\
$\mathrm{Cs}(1) / \mathrm{Rb}(1)-\mathrm{O}(1) \# 1$ & $3.1449(89)$ & $\mathrm{O}(2) \# 12-\mathrm{Al}(1)-\mathrm{F}(1)$ & $103.524(36)$ \\
$\mathrm{Cs}(1) / \mathrm{Rb}(1)-\mathrm{O}(1) \# 2$ & $3.1449(10)$ & $\mathrm{O}(2) \# 13-\mathrm{Al}(1)-\mathrm{F}(1)$ & $103.524(36)$ \\
$\mathrm{Cs}(1) / \mathrm{Rb}(1)-\mathrm{O}(1) \# 3$ & $3.1449(89)$ & $\mathrm{O}(2) \# 12-\mathrm{Al}(1)-\mathrm{O}(2) \# 13$ & $114.706(50)$ \\
$\mathrm{Cs}(1) / \mathrm{Rb}(1)-\mathrm{O}(1) \# 4$ & $3.1449(10)$ & $\mathrm{O}(2) \# 13-\mathrm{Al}(1)-\mathrm{O}(2)$ & $114.706(59)$ \\
$\mathrm{Cs}(1) / \mathrm{Rb}(1)-\mathrm{O}(1) \# 5$ & $3.1449(12)$ & $\mathrm{O}(2) \# 12-\mathrm{Al}(1)-\mathrm{O}(2)$ & $114.706(59)$ \\
$\mathrm{Cs}(1) / \mathrm{Rb}(1)-\mathrm{O}(2) \# 6$ & $3.2145(11)$ & $\mathrm{O}(1)-\mathrm{B}(1)-\mathrm{O}(1) \# 14$ & $118.003(16)$ \\
$\mathrm{Cs}(1) / \mathrm{Rb}(1)-\mathrm{O}(2) \# 7$ & $3.2145(13)$ & $\mathrm{O}(1)-\mathrm{B}(1)-\mathrm{O}(2) \# 15$ & $120.531(14)$ \\
$\mathrm{Cs}(1) / \mathrm{Rb}(1)-\mathrm{O}(2) \# 8$ & $3.2145(92)$ & $\mathrm{O}(1) \# 14-\mathrm{B}(1)-\mathrm{O}(2) \# 15$ & $120.120(21)$ \\
$\mathrm{Cs}(1) / \mathrm{Rb}(1)-\mathrm{O}(2) \# 9$ & $3.2145(13)$ & & \\
$\mathrm{Cs}(1) / \mathrm{Rb}(1)-\mathrm{O}(2) \# 10$ & $3.2145(92)$ & & \\
$\mathrm{Cs}(1) / \mathrm{Rb}(1)-\mathrm{O}(2) \# 11$ & $3.2145(11)$ & & \\
$\mathrm{Al}(1)-\mathrm{F}(1)$ & $1.860(15)$ & & \\
$\mathrm{Al}(1)-\mathrm{O}(2)$ & $1.667(11)$ & & \\
$\mathrm{Al}(1)-\mathrm{O}(2) \# 12$ & $1.6675(13)$ & & \\
$\mathrm{Al}(1)-\mathrm{O}(2) \# 13$ & $1.6675(85)$ & & \\
$\mathrm{B}(1)-\mathrm{O}(1)$ & $1.391(18)$ & & \\
$\mathrm{B}(1)-\mathrm{O}(1) \# 14$ & $1.3686(33)$ & & \\
$\mathrm{B}(1)-\mathrm{O}(2) \# 15$ & $1.3398(22)$ & & \\
\hline $\mathrm{S} y m m$ & & \\
\hline
\end{tabular}

Symmetry transformations used to generate equivalent atoms:

\#1 -1-y,1+x-y,z; \#2 -2-x+y, -1-x,z; \#3 x-y,-y,-1-z; \#4 -2-x,-1-x+y,-1-z; \#5 -1+y,1+x,-1-z; \#6 -1-x+y,-1-x,-3/2-z; \#7 $\quad$ x,1+y,-3/2-z; \#8 $\quad$-2-y,x-y,-3/2-z; \#9 y,1+x,1/2+z; $\# 10-1+\mathrm{x}-\mathrm{y},-1-\mathrm{y}, 1 / 2+\mathrm{z} ; \quad \# 11-2-\mathrm{x},-\mathrm{x}+\mathrm{y}, 1 / 2+\mathrm{z} ; \quad \# 12-2-\mathrm{y}, \mathrm{x}-\mathrm{y}, \mathrm{z} ; \quad \# 13-2-\mathrm{x}+\mathrm{y},-2-\mathrm{x}, \mathrm{z} ;$ $\# 14-1-\mathrm{y}, \mathrm{x}-\mathrm{y}, \mathrm{z} ; \quad \# 15 \mathrm{x}, \mathrm{y},-3 / 2-\mathrm{z}$ 

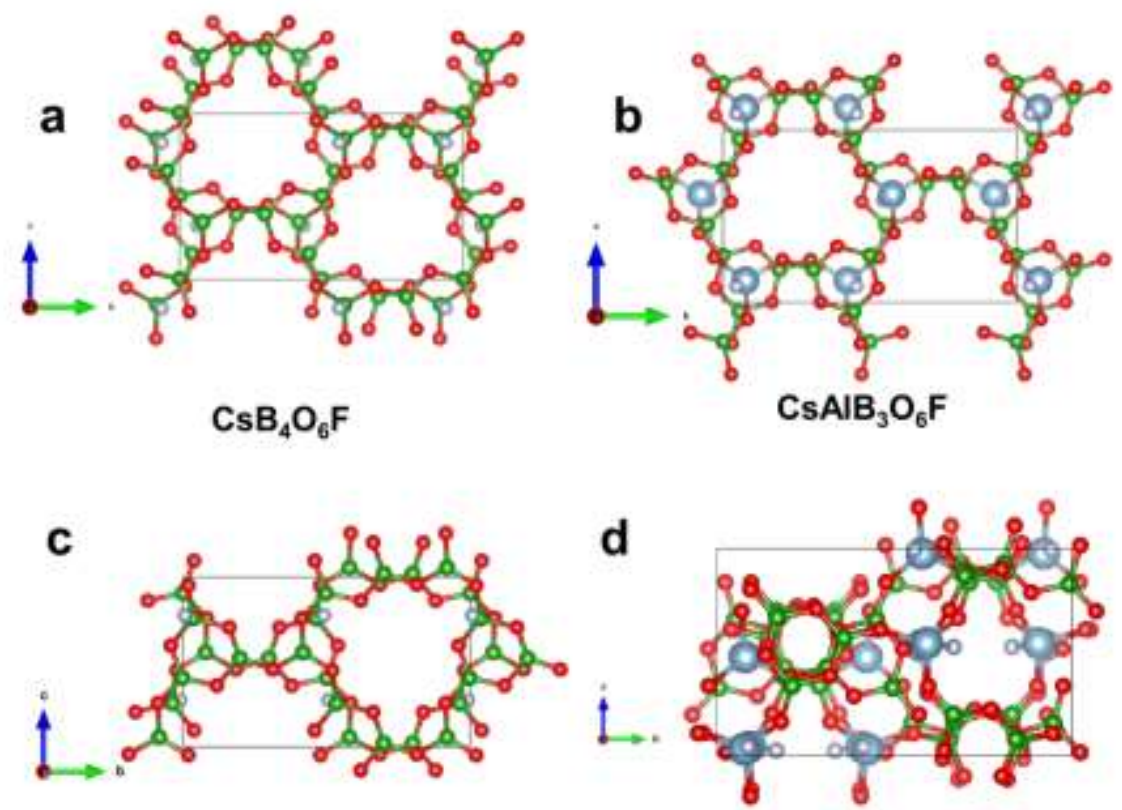

$\mathrm{RbB}_{4} \mathrm{O}_{6} \mathrm{~F}$

$\mathrm{RbAlB}_{3} \mathrm{O}_{6} \mathrm{~F}$

e

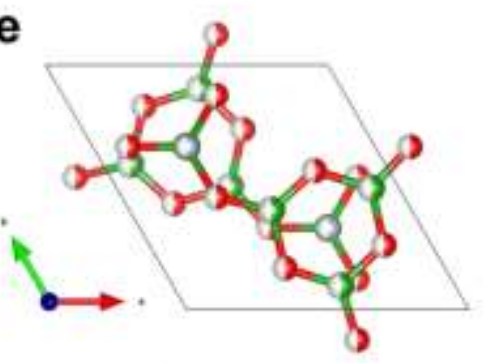

$\mathrm{Cs}_{0.5} \mathrm{Rb}_{0.5} \mathrm{~B}_{4} \mathrm{O}_{6} \mathrm{~F}$

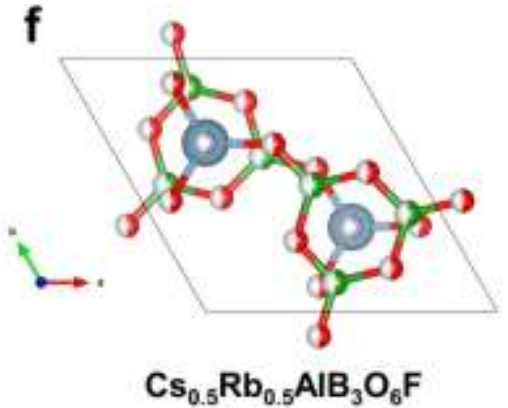

Figure S1. Crystal structure comparison of (a) $\mathrm{CsB}_{4} \mathrm{O}_{6} \mathrm{~F}$, (b) $\mathrm{CsAlB}_{3} \mathrm{O}_{6} \mathrm{~F}$, (c) $\mathrm{RbB}_{4} \mathrm{O}_{6} \mathrm{~F}$, (d) RABF, (e) $\mathrm{Cs}_{0.5} \mathrm{Rb}_{0.5} \mathrm{~B}_{4} \mathrm{O}_{6} \mathrm{~F}$, and (f) CRABF. 
a

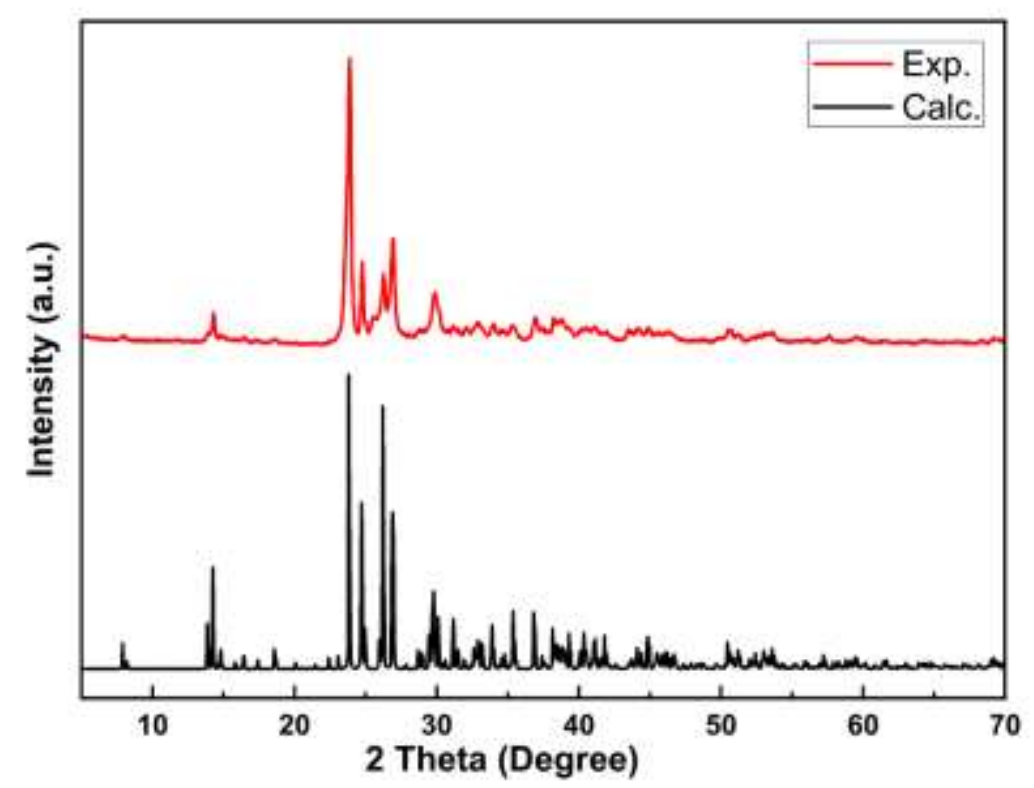

b

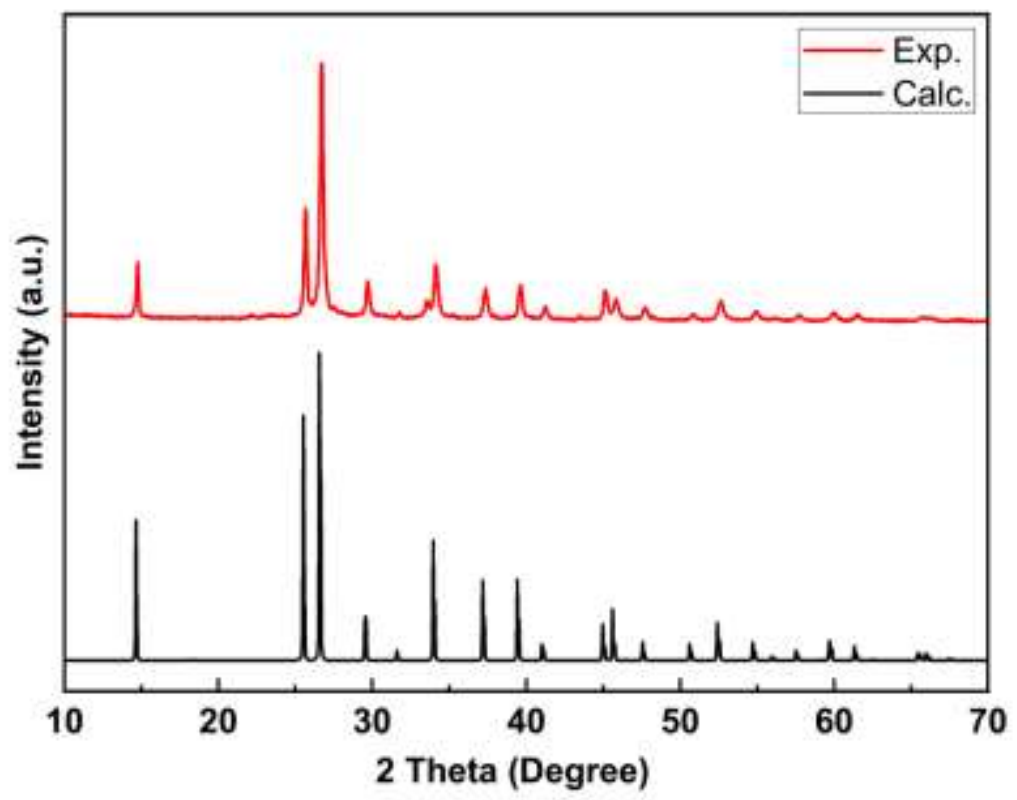

Figure S2. The calculated and experiment PXRD patterns of (a) RABF and (b) CRABF. 
a

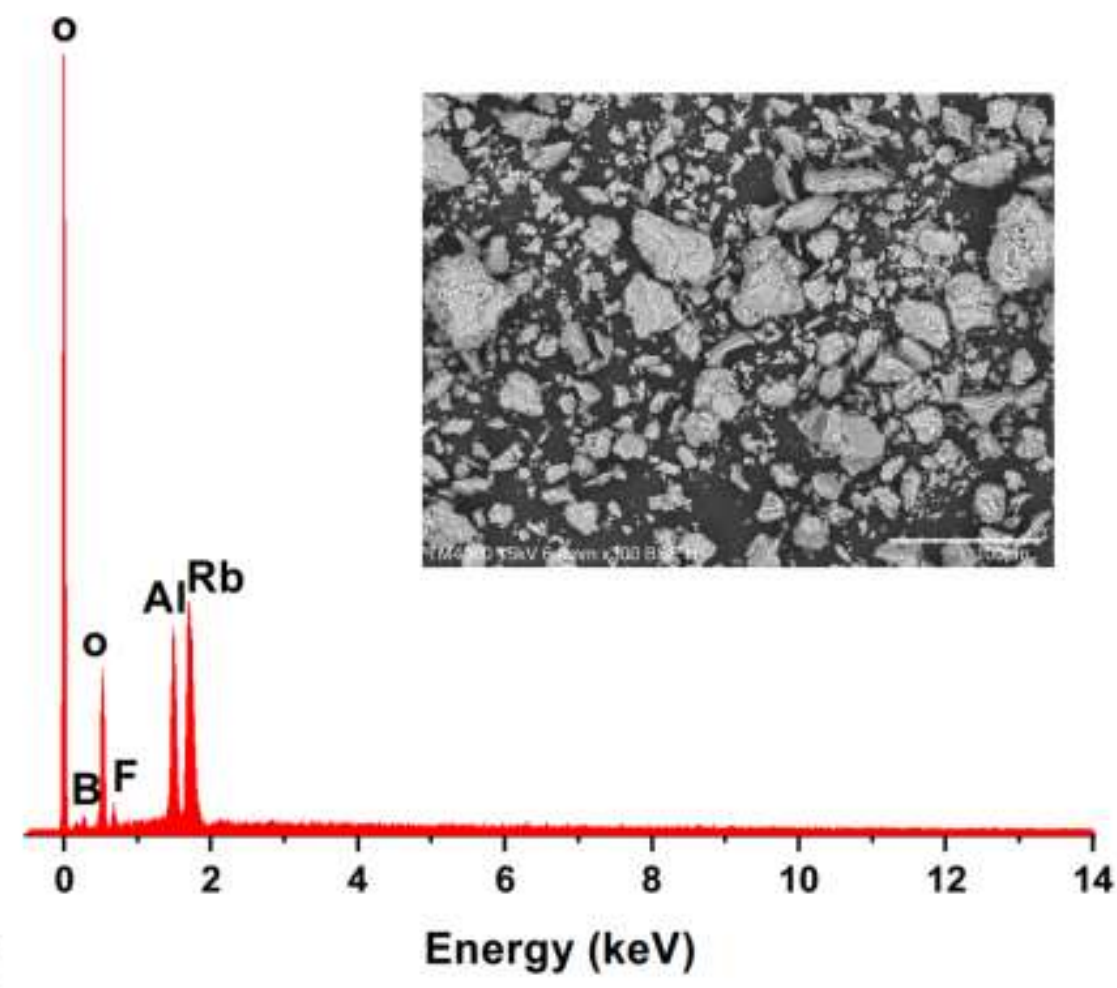

b Energy (keV)

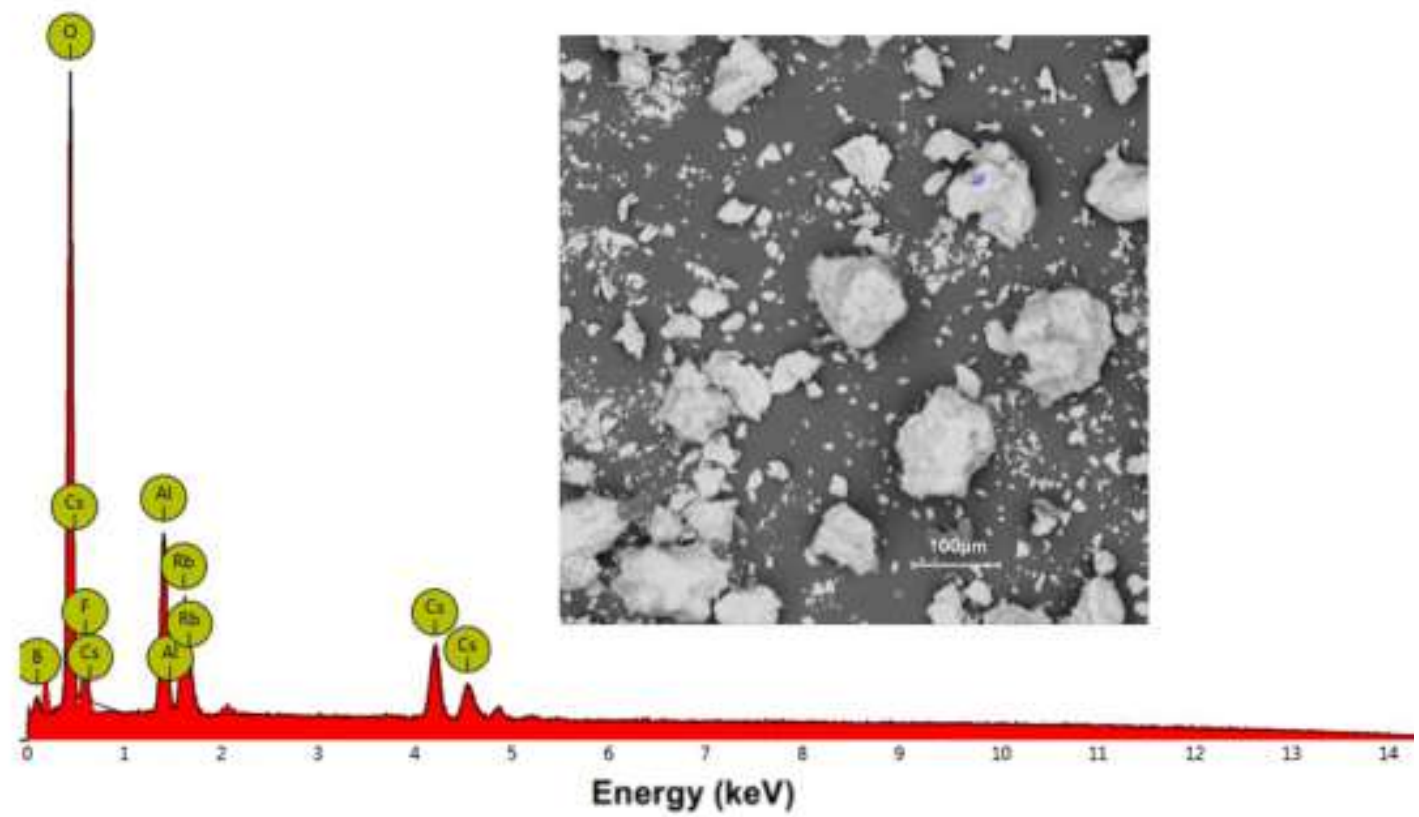

Figure S3. EDX spectroscopy and SEM image (insets) of (a) RABF and (b) CRABF. The EDX analysis indicates that the molar ratios of O: F are 52.43: 9.96 and 43.24:6.90, for RABF and CRABF, respectively, which approximately equal to the theoretical molar ratio of $6: 1$. The results further verify the existence of fluorine in the crystal structures. 


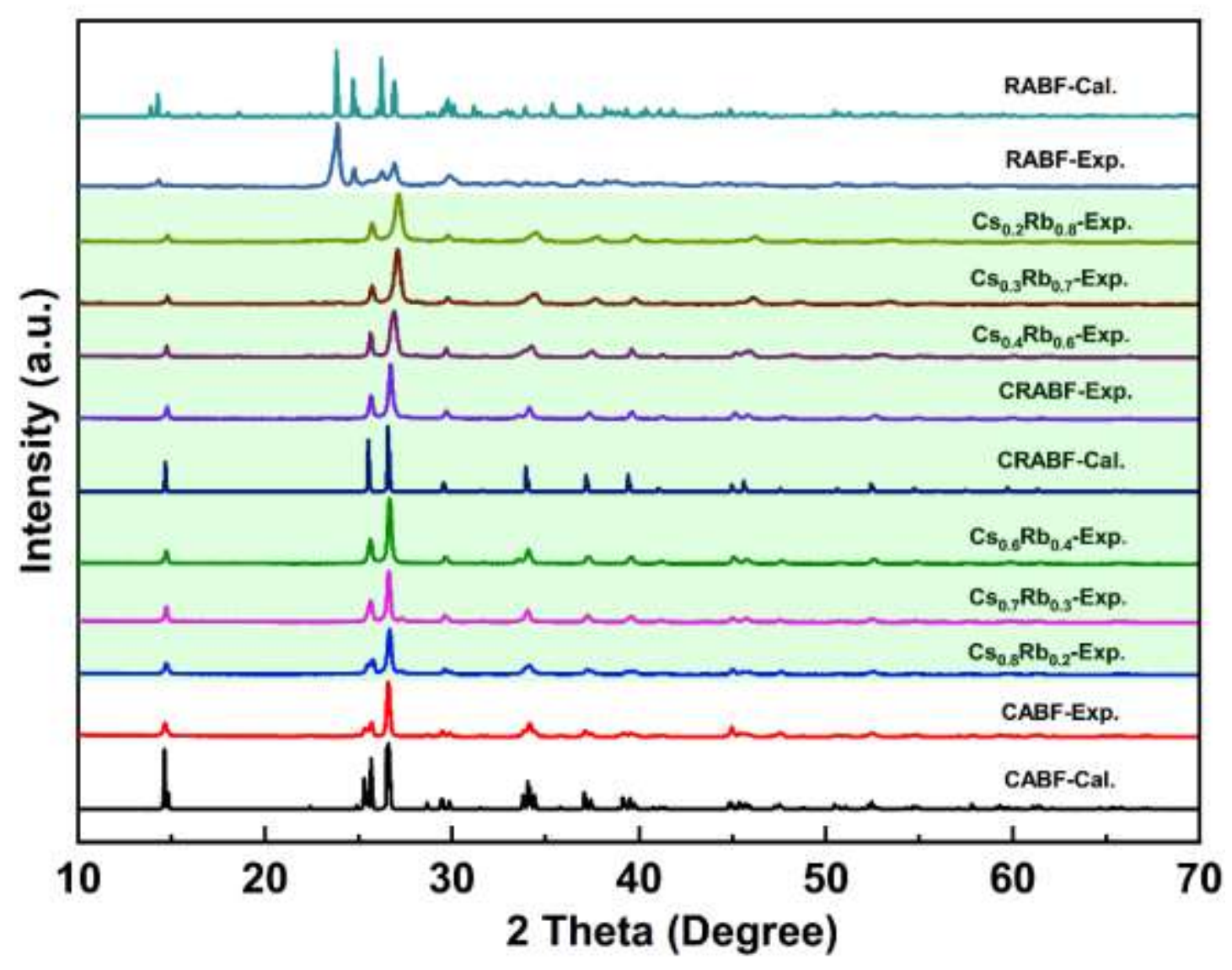

Figure S4. The calculated and experiment PXRD patterns of $\mathrm{Cs}_{x} \mathrm{Rb}_{1-x} \mathrm{AlB}_{3} \mathrm{O}_{6} \mathrm{~F}$ with different $x$ values. 
a

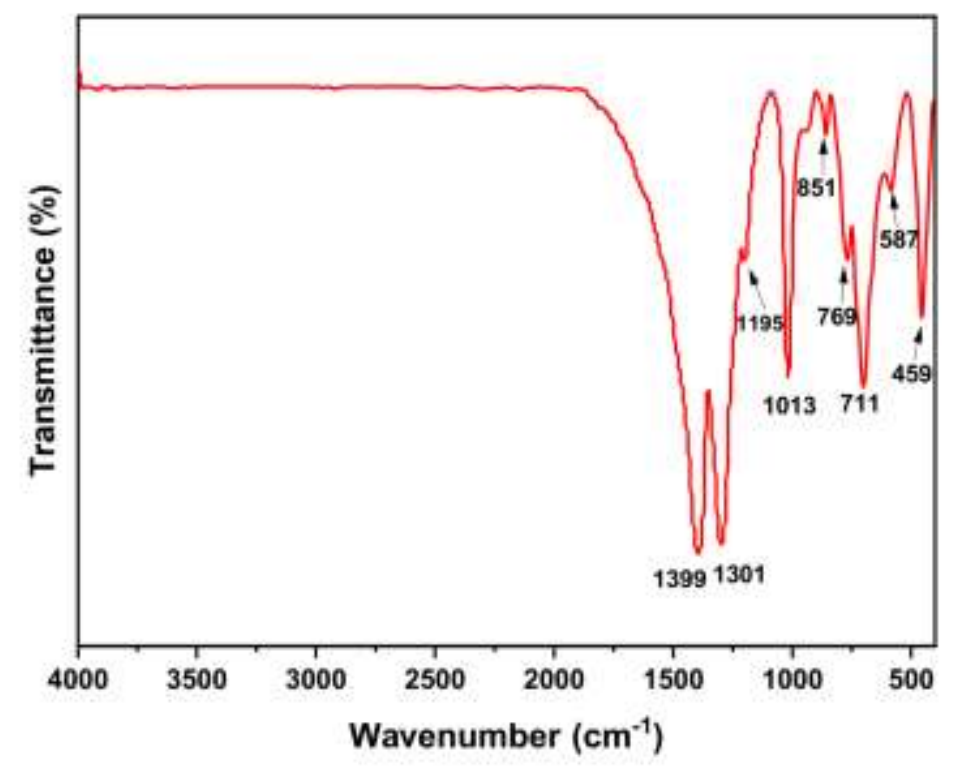

b

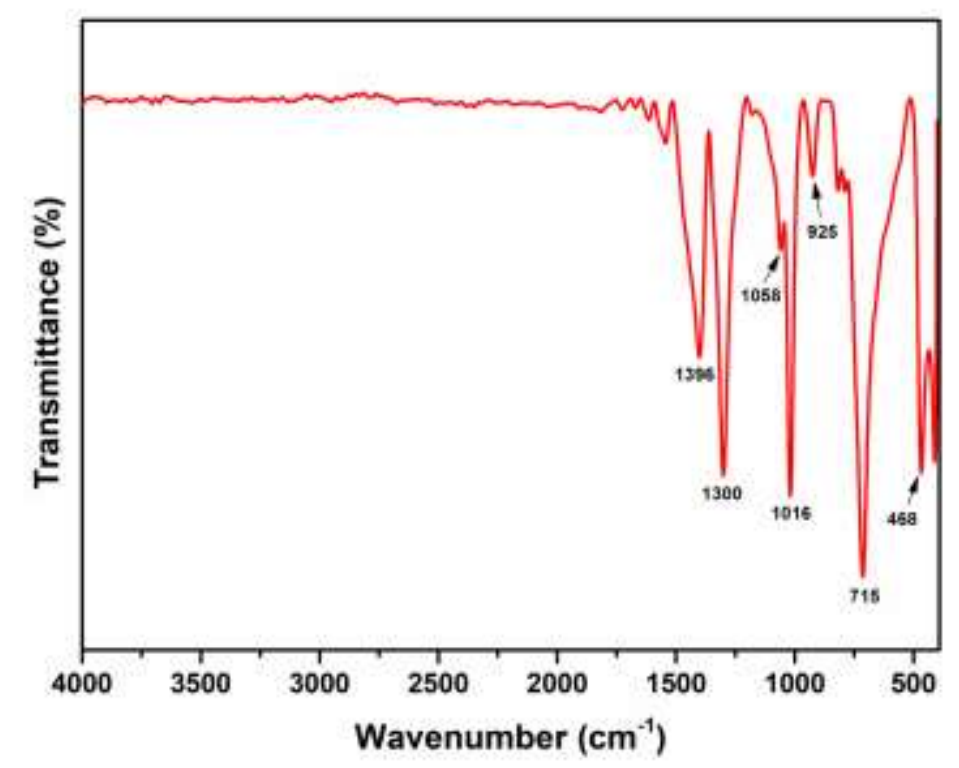

Figure S5. The IR spectrum of (a) RABF and (b) CRABF.

\begin{tabular}{ll}
\hline peaks & modes \\
\hline $1396,1399 \mathrm{~cm}^{-1}$ and $1300,1304 \mathrm{~cm}^{-1}$ & asymmetric stretching of the $\mathrm{BO}_{3}$ groups \\
$1058,1195 \mathrm{~cm}^{-1}$ and $1013,1016 \mathrm{~cm}^{-1}$ & symmetric stretching of the $\mathrm{BO}_{3}$ groups \\
$851 \sim 925 \mathrm{~cm}^{-1}$ & out-of-plane bending of the $\mathrm{BO}_{3}$ groups \\
$711,715 \mathrm{~cm}^{-1}$ and $459,468 \mathrm{~cm}^{-1}$ & stretching-bending vibrations of the $\mathrm{AlO}_{3} \mathrm{~F}$ units \\
\hline
\end{tabular}


a

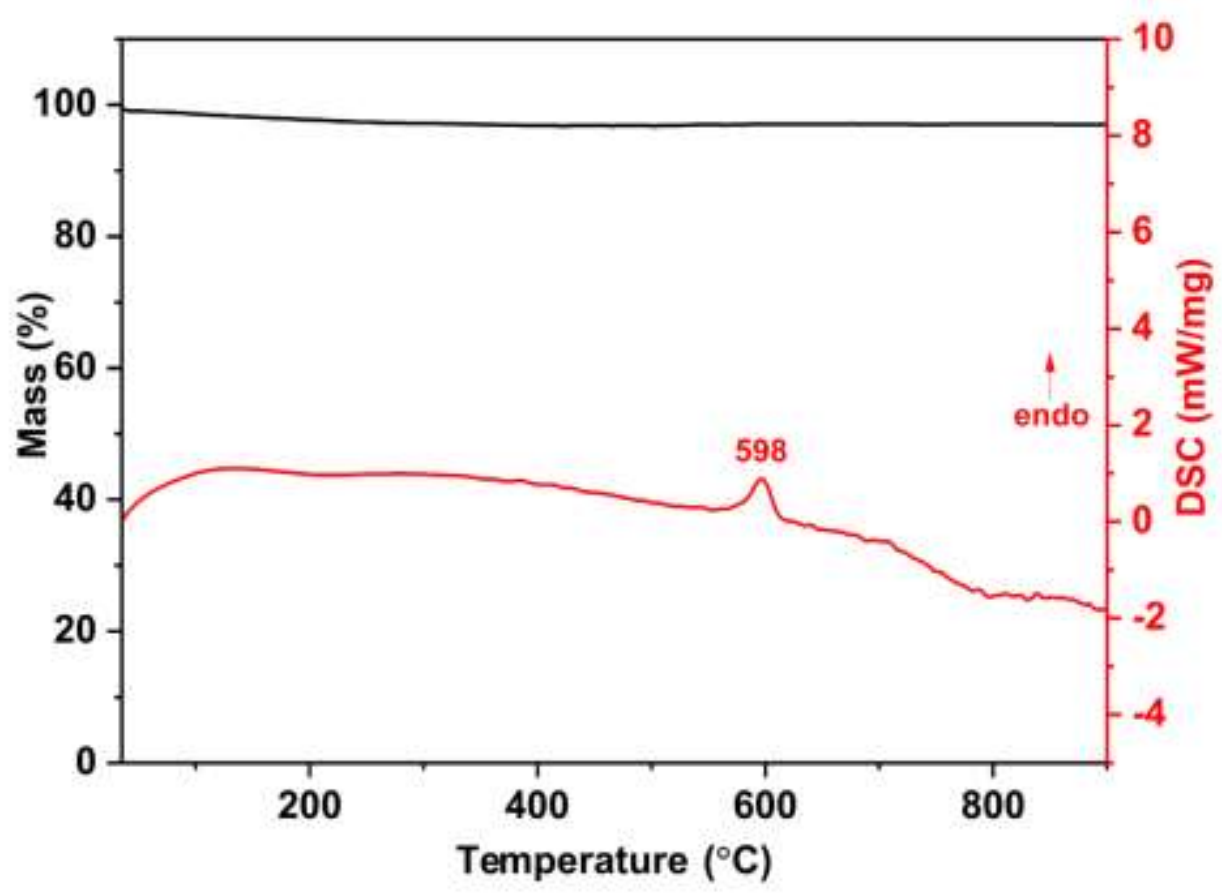

b

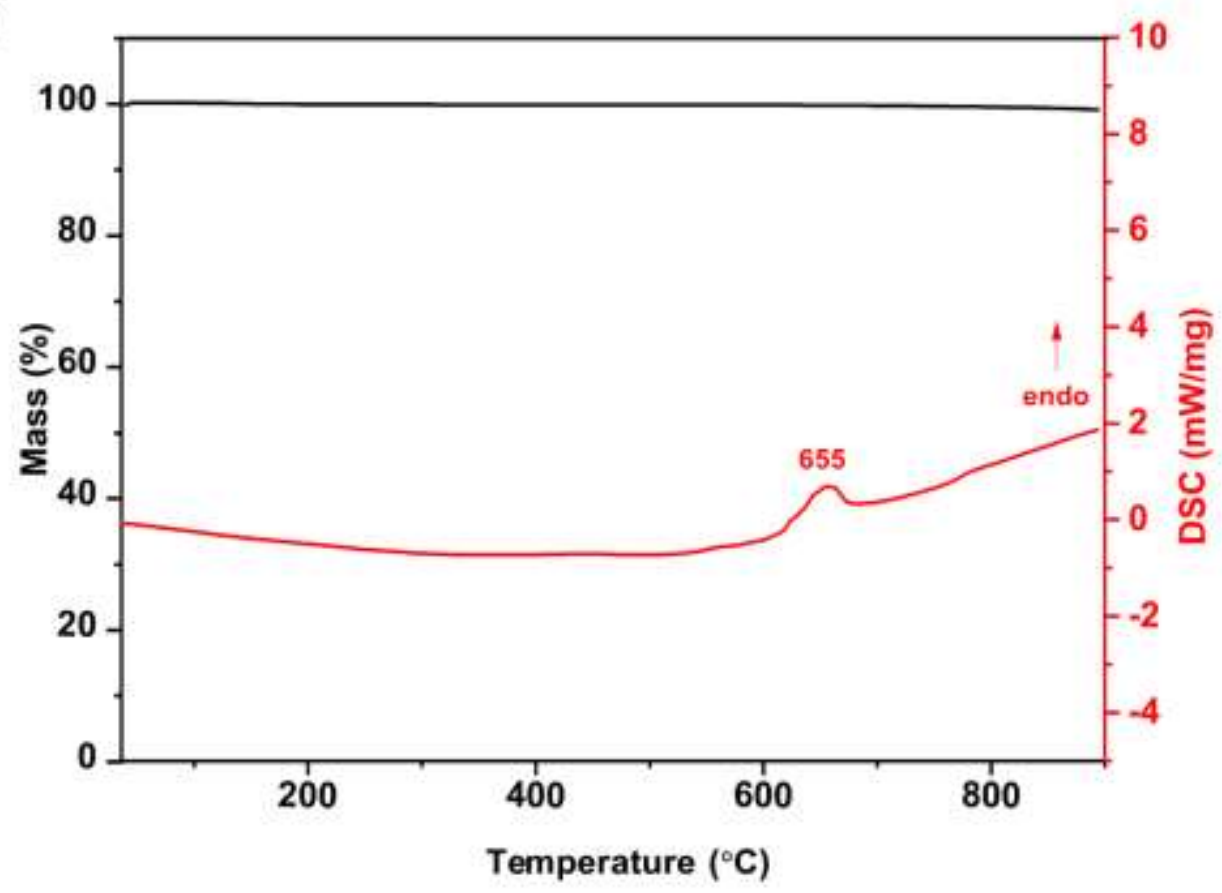

Figure S6. The TG/DSC curves of (a) RABF and (b) CRABF. 
a

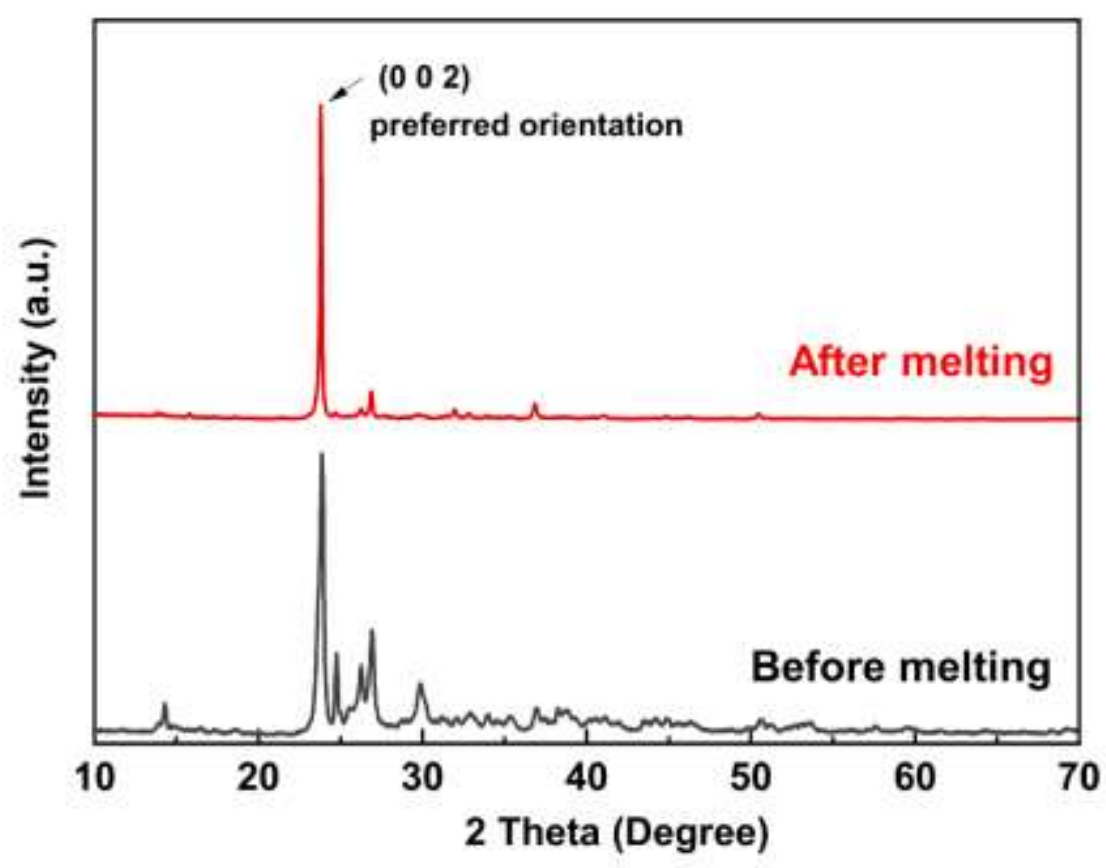

b

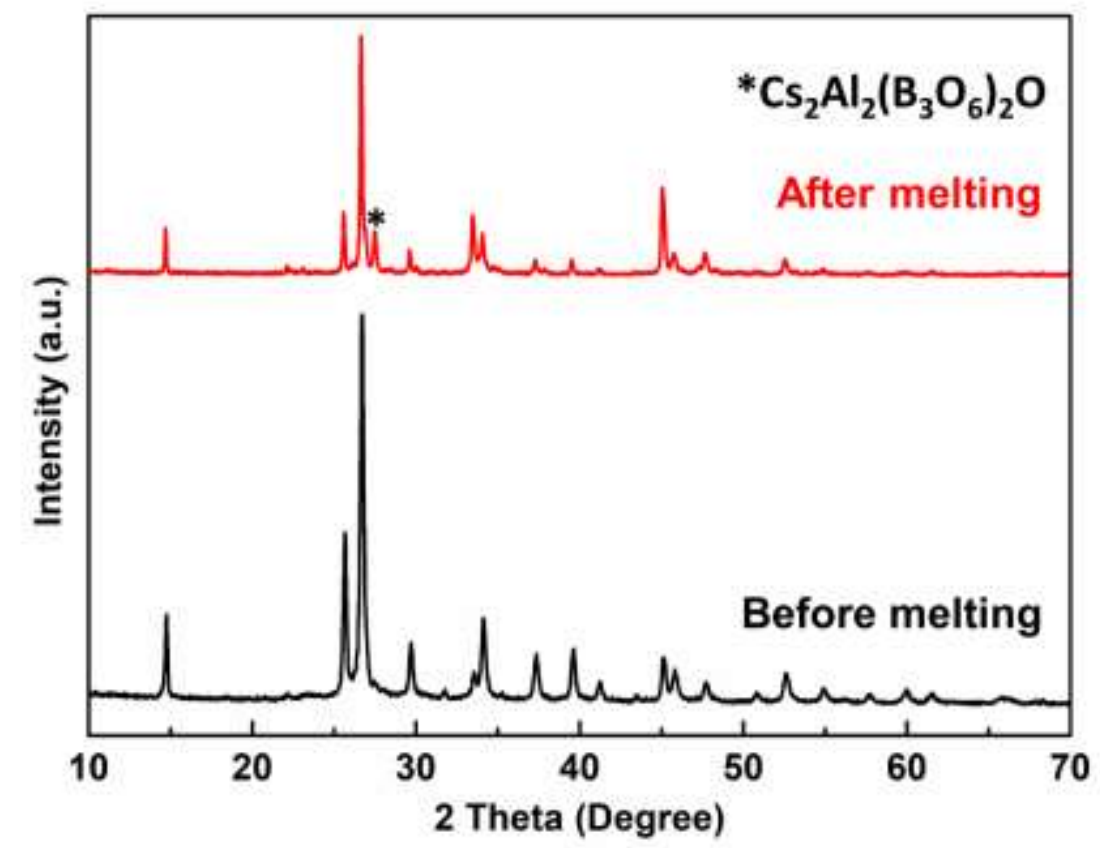

Figure S7. The XRD pattarns of before and after melting of (a) RABF and (b) CRABF. 
a

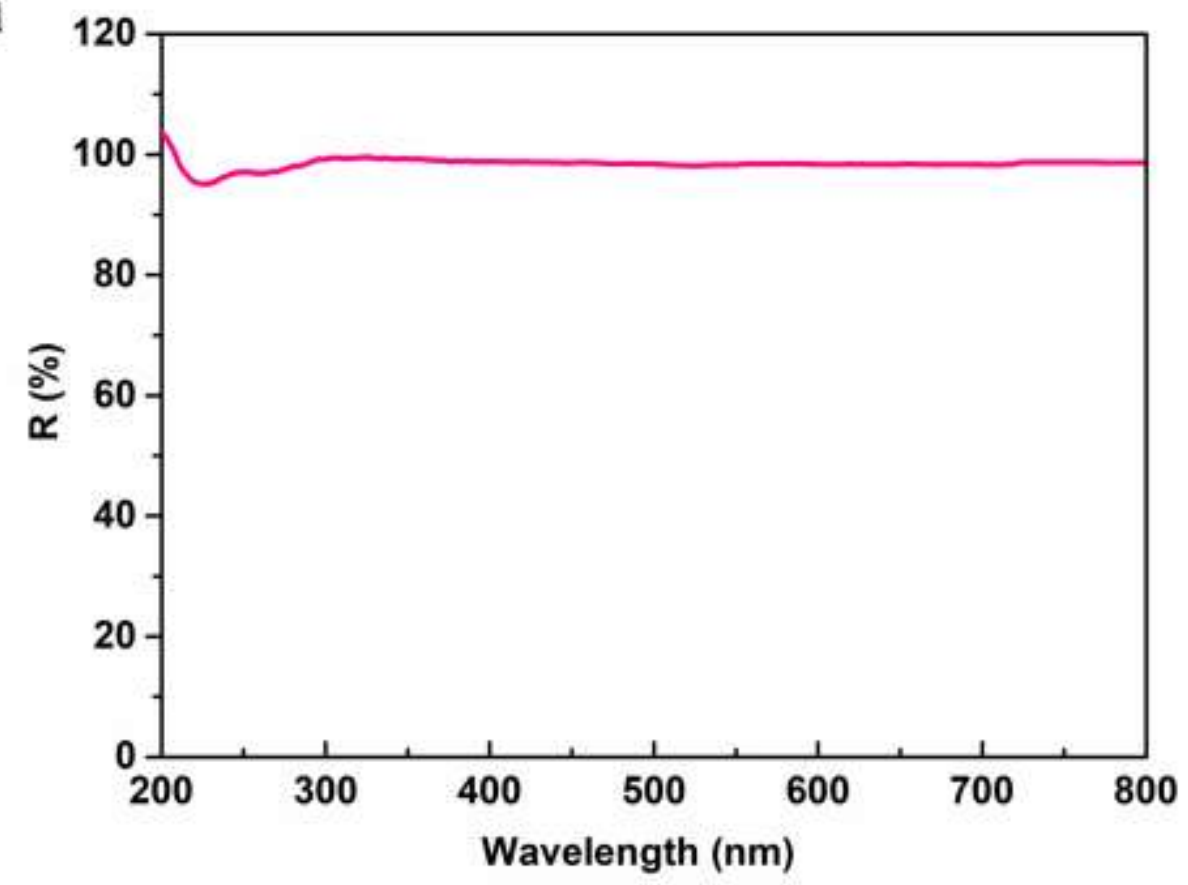

b

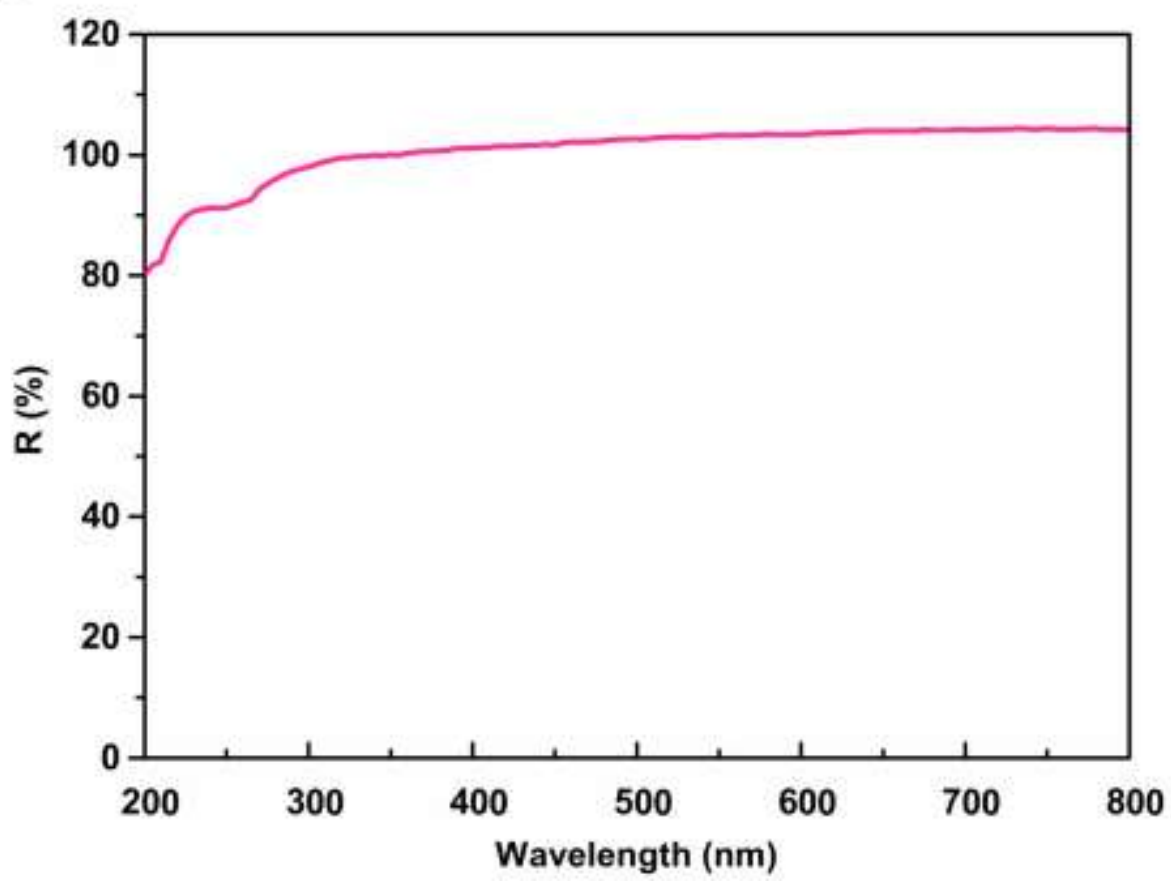

Figure S8. The diffuse reflectance spectrum of (a) RABF and (b) CRABF. 


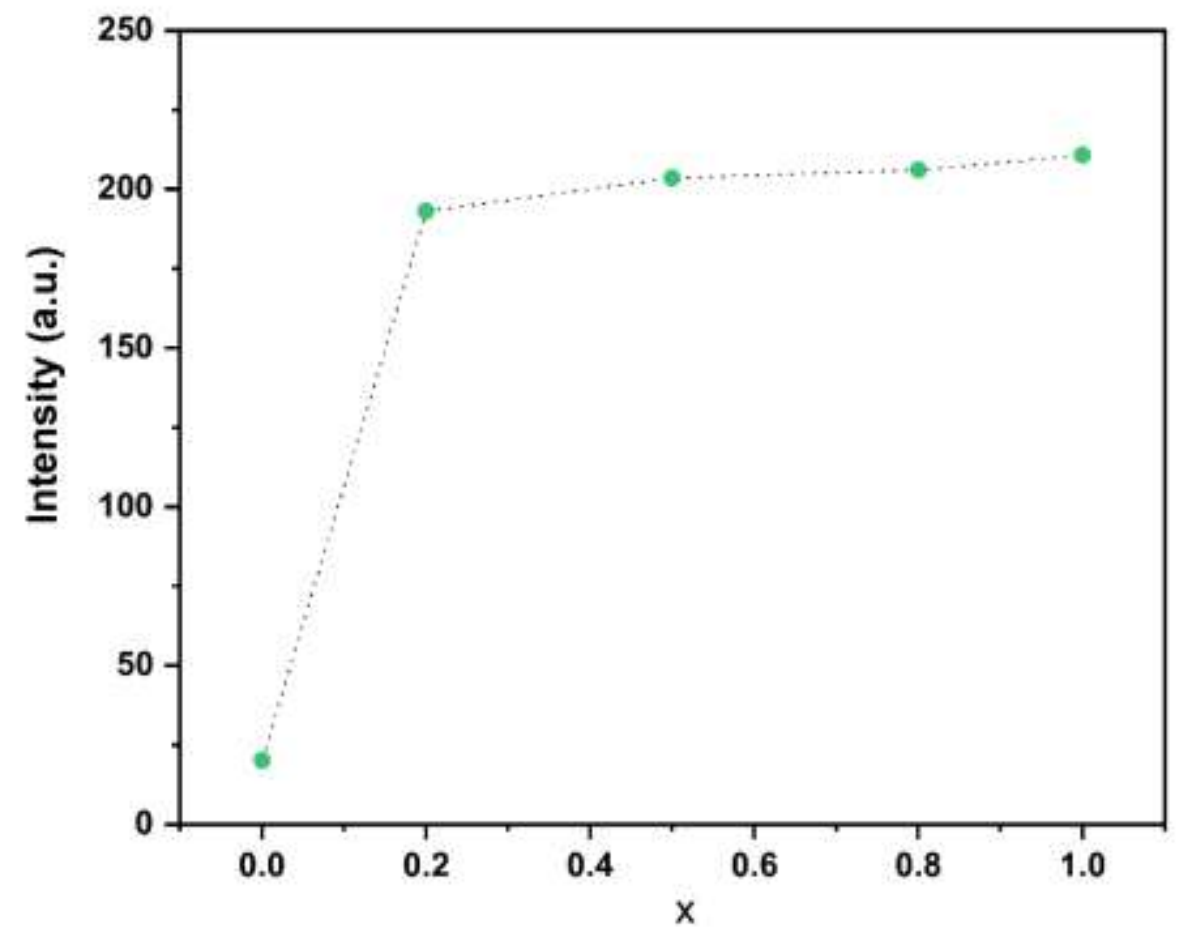

Figure S9. The SHG intensities of $\mathrm{Cs}_{x} \mathrm{Rb}_{1-x} \mathrm{AlB}_{3} \mathrm{O}_{6} \mathrm{~F}$ with different $x$ values. 

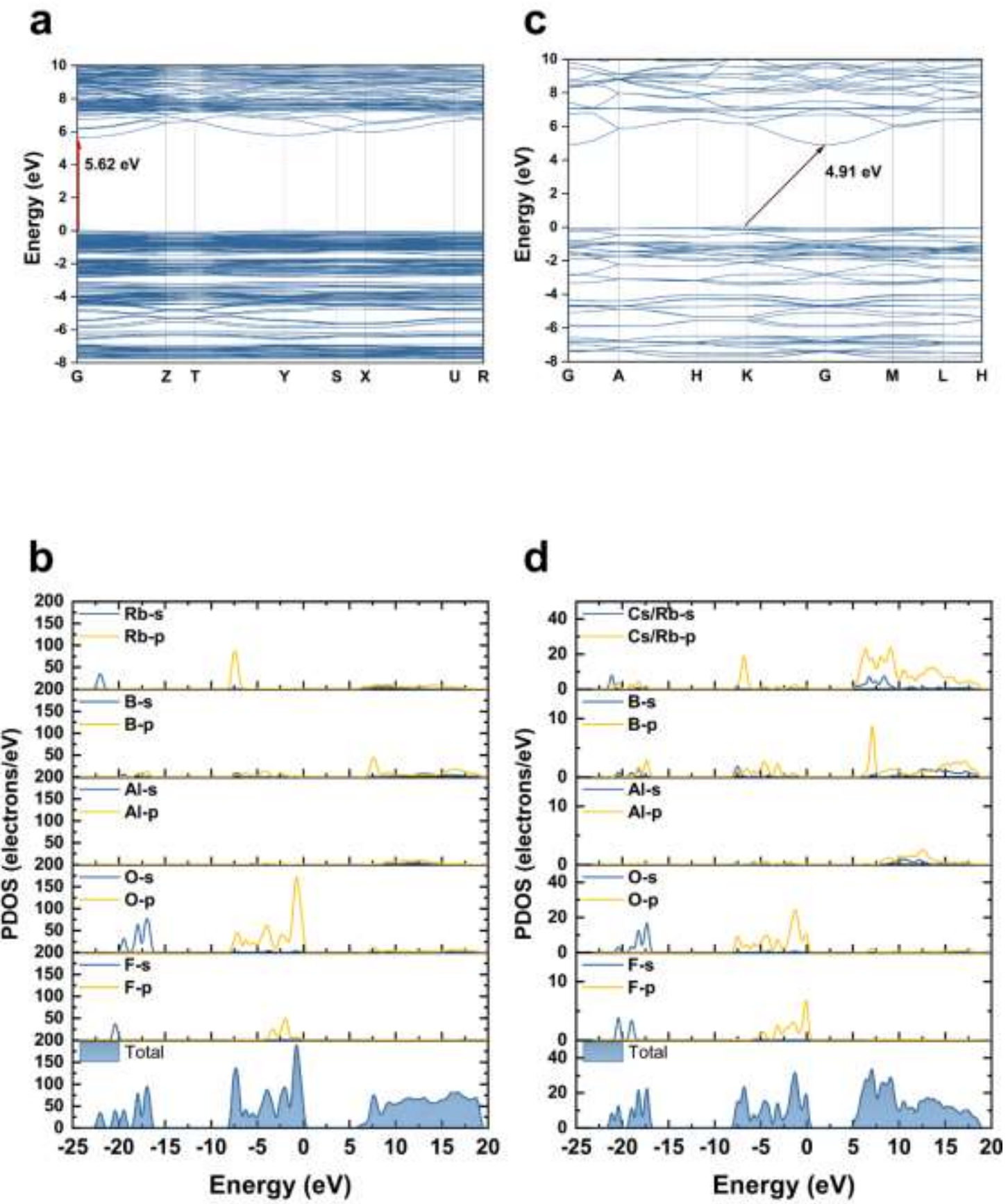

Figure S10. The band gap and PDOS of (a-b) RABF and (c-d) CRABF. 


\section{References}

(1). Yong, Z.; Chao, Y. Y.; Nuo, W. J.; Feng, Y.; Kun, C. X.; Fu, C. D.; Jun, P. Q.; Gui, H. Z.; Yan, X. Z., Nonlinear Optical Properties of $\mathrm{BaAlBO}_{3} \mathrm{~F}_{2}$ Crystal. Opt. Express 2009, 17, 20033-20038.

(2). Hu, Z. G.; Yue, Y. C.; Chen, X. A.; Yao, J. Y.; Wang, J. N.; Lin, Z. S., Growth and Structure Redetermination of a Nonlinear $\mathrm{BaAlBO}_{3} \mathrm{~F}_{2}$ Crystal. Solid State Sci. 2011, 13, 875-878.

(3). Wang, J. N.; Yue, Y. C.; Yao, J. Y.; Hu, Z. G., Growth and Characterization of Ba(Al,Ga)BO $\mathrm{B}_{3} \mathrm{~F}_{2}$ Crystal. J. Cryst. Growth 2011, 318, 962-965.

(4). Zhao, S. G.; Gong, P. F.; Luo, S. Y.; Liu, S. J.; Li, L.; Asghar, M. A.; Khan, T.; Hong, M. C.; Lin, Z. S.; Luo, J. H., Beryllium-Free $\mathrm{Rb}_{3} \mathrm{Al}_{3} \mathrm{~B}_{3} \mathrm{O}_{10} \mathrm{~F}$ with Reinforced Interlayer Bonding as a Deep-Ultraviolet Nonlinear Optical Crystal. J. Am. Chem. Soc. 2015, 137, 2207-2210.

(5). Liu, H. K.; Wang, Y.; Zhang, B. B.; Yang, Z. H.; Pan, S. L., CsAlB ${ }_{3} \mathrm{O}_{6}$ F: A Beryllium-Free Deep-Ultraviolet Nonlinear Optical Material with Enhanced Thermal Stability. Chem. Sci. 2020, 11, 694-698. 